\title{
Three Dimensional Visualization of Electromagnetic Fields from One Dimensional Nanostructures
}

\author{
Charudatta Phatak ${ }^{1}$, Aurélien Masseboeuf ${ }^{2}$, Ludvig de Knoop ${ }^{2}$, Christophe Gatel ${ }^{2,3}$, Martin J. Hÿtch $^{2}$ \\ ${ }^{1}$ Materials Science Division, Argonne National Laboratory, Lemont, IL 60439, USA \\ ${ }^{2}$ CEMES-CNRS, 29, rue Jeanne Marvig, FR - 31055 Toulouse, France \\ ${ }^{3}$ Université Paul Sabatier, F-31000 Toulouse, France.
}

One-dimensional (1D) nanostructures have been regarded as the most promising building blocks for nanoelectronics and nanocomposite material systems as well as for alternative energy applications [1]. Magnetic nanowires with circular cross-section, are of utmost importance from theoretical and technological aspects [2]. 1D carbon-based nanostructures such as carbon nanotubes are amongst the best candidates for field emission displays and new high-brightness electron sources [3]. The confinement effects in 1D nanostructures can alter their properties and subsequently their behavior significantly. Hence it is necessary to understand the strong effect of their size on their three-dimensional (3D) properties such as the magnetic and electric fields associated with nanowires and nanotubes completely before they can be used in applications. There are currently very few methods, which have the capability to visualize the complete $3 \mathrm{D}$ fields associated with nanowires. In this work, we show that using a combination of symmetry arguments and electron-optical phase shift data obtained using TEM, it is possible to recover the entire 3D magnetic or electric field in and around nanowires and nanotubes from a single image.

The phase change $\varphi_{e}+\varphi_{m}$ of an electron wave traveling through a thin foil can be expressed in terms of tomographic quantities [4]. The phase shift can be recovered experimentally using various techniques such as transport-of-intensity based methods or off-axis electron holography. Nominally, determining the 3D magnetic field or 3D elestrostatic potential requires recording a series of phase shift images as the sample is tilted about its axis. However, in a 1D nanostructure such as a nanocylinder that is uniformly magnetized along its long axis, the magnetic field possesses cylindrical symmetry with respect to the long axis. Similarly, carbon nanotubes under applied bias exhibit a cylindrically symmetric potential and electric field. Exploiting these conditions of cylindrical symmetry, we can reconstruct the full 3D magnetic or electric field associated with a nanowire from a single phase image using the inverse Abel transform. Simulations were performed for a uniformly magnetized sphere, which also possesses cylindrical symmetry to analyze the fidelty of the reconstruction algorithm. Fig. 1(a) shows the magnetic phase shift of such a uniformly magnetized sphere, (b) shows the derivative with respective to the horizontal axis $\left(\partial \varphi_{m} / \partial x\right)$ showing the cylindrical symmetry, and (c) the 3D reconstructed magnetic induction using the single image method developed in this work.

Experiments were performed on nickel nanowires that were uniformly magnetized to reconstruct the 3D magnetic induction. Similarly the 3D electric field was reconstructed from in-situ biased carbon cone nanotips under varying applied bias. The phase shift was recovered using off-axis electron holography in a FEI Tecnai F20 TEM operating at $200 \mathrm{kV}$ using the first transfer lens of the Cs-corrector as a Lorentz lens. The magnetic phase shift was obtained from the Ni nanowire by recording the phase shift images with the sample as-is and rotating it by $180^{\circ}$ about its axis and then subtracting the two 
phase shifts. Fig. 2 (a) shows the magnetic phase shift of the Ni nanowire and (b) shows the reconstructed 3D magnetic induction. We will further discuss the details of the application and limitation of this method to reconstruct the 3D electromagnetic fields.

\section{References}

[1] C. M. Lieber, Solid State Communications, 107, 607 (1998).

[2] C. Chappert, A. Fert, F. N. Van Dau, Nature Materials, 6, 813 (2007).

[3] N. de Jonge, Y. Lamy, K. Schoots, T. H. Oosterkamp, Nature, 420, 393 (2002).

[4] C. Phatak, M. Beleggia, and M. De Graef, Ultramicroscopy, 108, 503 (2008).

[5] This work was support by U.S. Department of Energy (DOE), Office of Science, Materials Sciences and Engineering Division.

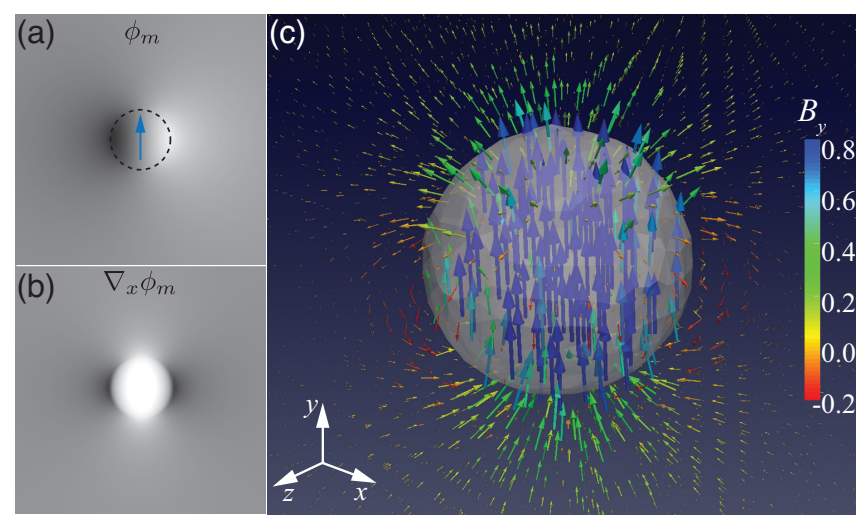

Figure 1: (a) shows the magnetic phase shift image of a uniformly magnetized spherical nanoparticle, indicated by the dashed line, and magnetization vector is indicated by the blue arrow, (b) the derivative of the magnetic phase shift with respect to $x$ showing the symmetry about the vertical axis of the image, and (c) the 3D reconstructed magnetic induction.
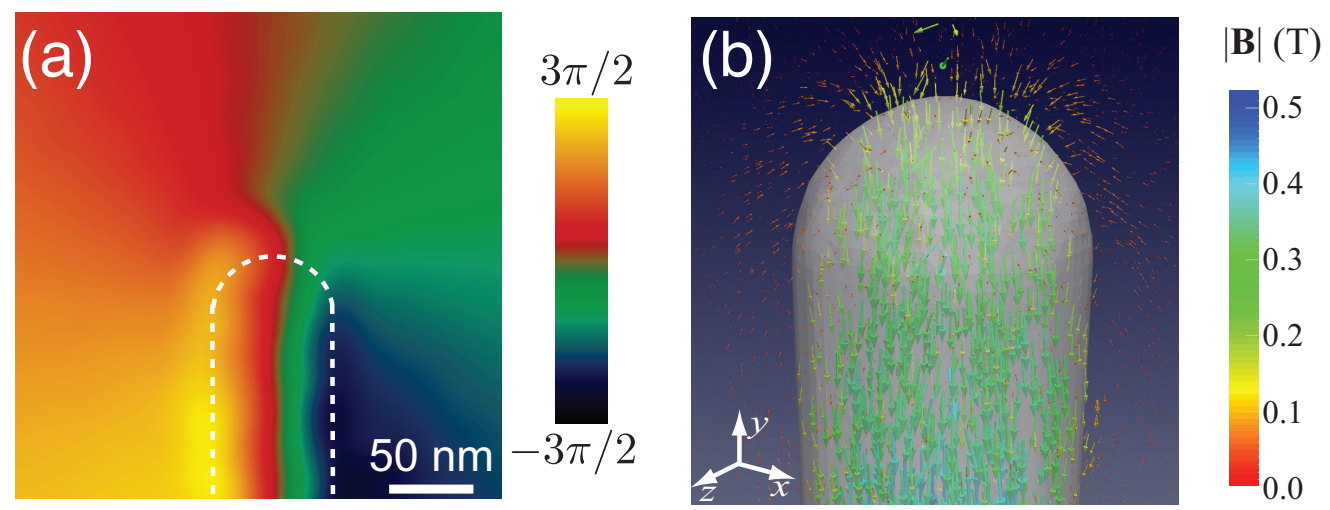

Figure 2: (a) shows the experimentally recovered magnetic phase shift from a Ni nanowire. The dotted lines indicate the border of the nanowire. (b) the 3D reconstructed magnetic induction from the nanowire. 